\title{
PERFIL SEMIOLÓGICO DE PACIENTES ACOMETIDOS POR ACIDENTE VASCULAR ENCEFÁLICO
}

\section{SEMIOLOGICAL PROFILE OF PATIENTS AFFECTED BY BRAIN VASCULAR ACCIDENT}

\author{
Natalia Tavares Sampaio ${ }^{1}$ \\ José Aurélio de Oliveira Figueiredo² \\ Juliane Carla Medeiros de Sousa ${ }^{3}$ \\ Luciano Braga de Oliveira ${ }^{4}$ \\ Michel Jorge Dias ${ }^{5}$
}

RESUMO: Objetivo: Conhecer o perfil semiológico de pacientes com diagnóstico de acidente vascular encefálico. Método: Trata-se de um estudo retrospectivo, realizado através da análise de prontuários, de forma descritiva, com abordagem quantitativa, desenvolvida nos meses de março e abril de 2019. O estudo foi composto por cinquenta prontuários de pacientes com diagnóstico de acidente vascular encefálico que foram atendidos entre os anos de 2013 a 2017 . Inclusos os pacientes de ambos os sexos, de todas as idades, cadastrados no setor de fisioterapia neurofuncional da clínica Santa Maria, e como critérios de exclusão, temse os prontuários incompletos, ilegíveis ou que o termo de consentimento não estivesse assinado pelo paciente ou responsável. $O$ instrumento de coleta de dados foi adaptado a partir da ficha de avaliação neurofuncional da clínica escola, onde foram abordados os itens do perfil socioeconômico, diagnóstico clínico, exames complementares, exame físico: tônus muscular, sensibilidade (superficial e profunda), reflexos, coordenação, equilíbrio, marcha, linguagem, força muscular e amplitude de movimento. Foi empregada estatística descritiva com realização de cálculos de frequência absoluta (n) e relativa (\%), medidas de tendência central (média), sendo os resultados apresentados em forma de tabelas e figuras, seguindo os aspectos éticos que envolvem a pesquisa com seres humanos. Resultados:

\footnotetext{
${ }^{1}$ Fisioterapeuta graduada pela Faculdade Santa Maria, Cajazeiras, PB.

${ }^{2}$ Fisioterapeuta. Mestre em Saúde Coletiva pela Universidade de Santos, Santos, SP. Docente da Faculdade Santa Maria, Cajazeiras, PB.

3 Fisioterapeuta. Mestre em Saúde Coletiva pela Universidade de Santos, Santos, SP. Docente da Faculdade Santa Maria, Cajazeiras, PB.

${ }^{4}$ Fisioterapeuta. Mestre em Saúde Coletiva pela Universidade de Santos, Santos, SP. Docente da Faculdade Santa Maria, Cajazeiras, PB.

5 Fisioterapeuta. Mestre em Saúde Coletiva pela Universidade de Santos, Santos, SP. Docente da Faculdade Santa Maria, Cajazeiras, PB. E-mail: michelj_dias@hotmail.com.
} 
Observou-se em relação ao perfil sociodemográfico a prevalência do sexo masculino com $56 \%$, faixa etária de 50 a 70 anos com $60 \%$, casados $(70 \%)$, ensino fundamental completo com 40\%. Em relação ao perfil semiológico foram encontradas como prevalentes: comprometimento do tipo isquêmico (92\%), tônus hipotônico $(60 \%)$, hipotrofismo $(18 \%)$, hipoestesia $(22 \%)$, hiporreflexia $(30 \%)$, alteração de coordenação (54\%), equilíbrio dinâmico (34\%), alteração da marcha $(84 \%)$ e linguagem (26\%). Conclusão: $O$ estudo evidenciou 0 maior comprometimento de acidente vascular encefálico do tipo isquêmico, sendo as alterações da marcha, tônus hipotônico e coordenação motora as alterações semiológicas mais prevalentes.

Descritores: Acidente vascular encefálico. Manifestações clínicas. Neurologia.

ABSTRACT: Objective: To know the semiological profile of patients diagnosed with stroke. Method: This is a retrospective study, carried out through the analysis of medical records, in a descriptive manner, with a quantitative approach, developed in the months of March and April 2019. The study consisted of fifty medical records of patients diagnosed with stroke that were attended between the years 2013 to 2017. Including patients of both sexes, of all ages, registered in the neurofunctional physiotherapy sector of the Santa Maria clinic, and as exclusion criteria, there are incomplete, illegible or that the consent form was not signed by the patient or guardian. The data collection instrument was adapted from the neurofunctional assessment form of the school clinic, where the items of the socioeconomic profile, clinical diagnosis, complementary exams, physical examination were addressed: muscle tone, sensitivity (superficial and deep), reflexes, coordination balance, gait, language, muscle strength and range of motion. Descriptive statistics were used with calculations of absolute ( $n$ ) and relative (\%) frequency, measures of central tendency (average), the results being presented in the form of tables and figures, following the ethical aspects that involve research with human beings. Results: It was observed in relation to the sociodemographic profile the prevalence of male sex with $56 \%$, age group from 50 to 70 years old with $60 \%$, married (70\%), complete elementary school with $40 \%$. Regarding the semiological profile, the following were found to be prevalent: ischemic type impairment (92\%), hypotonic tone (60\%), hypotrophism $(18 \%)$, hypoesthesia (22\%), hyporeflexia (30\%), change in coordination (54\%), dynamic balance (34\%), change in gait (84\%) and language (26\%). Conclusion: The study showed the greatest impairment of ischemic stroke, with changes in gait, hypotonic tone and motor coordination being the most prevalent semiological changes.

Descriptors: Encephalic Vascular Disease. Clinical Findings. Neurology. 


\section{INTRODUÇÃO}

Atualmente é possível observar que a população brasileira está vivenciando um estilo de vida contemporâneo cada vez mais vulnerável às doenças crônicas não transmissíveis (DCNT), correspondendo as mais relevantes causas de mortalidades, das quais, o acidente vascular encefálico ( $A V E$ ) é citado como uma das principais, caracterizando-se como de maior prevalência na população brasileira (FERNANDES et al., 2012).

O índice de AVE vem avultando de forma significativa, consolidando-a como problema de saúde pública devido ao grande número de acometimentos, bem como sendo considerada a patologia que mais gera incapacidades e óbitos (CARO; CRUZ, 2017), ocorrendo, principalmente, após os sessenta e cinco anos de idade (SÁ, GRAVE e PÉRICO, 2014).

Segundo a Organização Mundial de Saúde (OMS), estima-se que 15 milhões de pessoas são atingidas por essa doença a cada ano, dessas, cinco milhões ficam incapacitadas e outras cinco milhões vão a óbito (GADELHA, 2016), o que vem a acarretar impacto considerável nos custos em saúde pública (SÁ, GRAVE e PÉRICO, 2014).

Essa afecção pode ser conceituada como uma alteração na função neurológica, ocorrendo, principalmente, quando o fluxo sanguíneo sofre modificações, privando o suprimento de oxigênio e nutrientes, de modo a danificadas áreas do cérebro, podendo ser caracterizada como focal ou difusa (LEITE, NUNES e CORREA, 2011).

Existem inúmeros comprometimentos, dentre eles, as alterações motoras, que podem influenciar no tônus e postura, além de causar alterações na comunicação, nas quais as afasias, apraxias e disartrias são mais presentes. $O$ tamanho da lesão vai depender do local de acometimento no indivíduo, o que varia muito de um indivíduo para o outro, podendo, na maioria das vezes, deixá-los com inúmeras sequelas e completamente dependentes (LACERDA et al., 2018). 
No que se refere à qualidade de vida dos indivíduos acometidos pelo AVE, é possível observar uma restrição da cognição e função física, deixando-os incapacitados e limitados. Salientando que são pessoas com comprometimentos que desencadeiam dependências em quase todas suas funções, as situações podem provocar conflitos familiares que envolvem a incapacidade na realização das atividades ativamente (DELBONI; MALENGO; SCHMIDT, 2010).

Dessa forma, este estudo teve como objetivo conhecer o perfil semiológico de pacientes com diagnóstico de AVE, mostrando-se relevante no sentido de permitir traçar um perfil clínico que discorre acerca das diferenças enquanto alterações singularizadas, que podem ser distintas de indivíduo para indivíduo, possibilitando ampliação do conhecimento, além de ser consolidado como base para novas pesquisas. Importante considerar que os estudos epidemiológicos proporcionam a compreensão dos fenômenos que englobam a saúde da população, favorecendo um planejamento e elaboração de intervenções mais coerentes.

\section{MÉTODO}

Trata-se de um estudo retrospectivo, descritivo, com abordagem quantitativa, realizado através da análise de prontuários. A pesquisa foi realizada na clínica Santa Maria no período compreendido entre os meses de março e abril de 2019.

A amostra foi composta por cinquenta prontuários de pacientes com diagnóstico de AVE, atendidos entre os anos de 2013 a 2017, sendo inclusos os pacientes de ambos os sexos, de todas as idades, cadastrados no setor de fisioterapia neurofuncional da clínica escola. Como critérios de exclusão: prontuários incompletos, ilegíveis, ou que o termo de consentimento não estivesse assinado pelo paciente ou responsável.

O instrumento de coleta de dados foi adaptado a partir da ficha de avaliação neurofuncional da clínica escola, sendo abordados os itens do perfil socioeconômico (sexo, idade, escolaridade, estado civil e ocupação), diagnóstico clínico, exames complementares (ressonância magnética, tomografia computadorizada), exame 
físico (tônus muscular, sensibilidade - superficial e profunda, reflexos, coordenação, equilíbrio, marcha, linguagem, força muscular e amplitude de movimento).

A coleta de dados teve início após a aprovação pelo Comitê de Ética em Pesquisa da Faculdade Santa Maria (CEP-FSM), através do parecer número 3.198.039. O estudo seguiu os aspectos éticos que envolvem a pesquisa com seres humanos, e seguiu as normas da resolução 466/12 do Conselho Nacional de Saúde - Pesquisa envolvendo seres humanos (BRASIL, 2012).

A pesquisa foi realizada em dias alternados, nos turnos da manhã e tarde, nos horários que não coincidiam com as atividades acadêmicas. Obteve-se uma média de vinte prontuários analisados por dia, de modo que a coleta foi executada no período de três semanas.

Os dados coletados foram analisados com a utilização do software Microsoft Office Excel (versão 2013), sendo empregada a estatística descritiva com realização de cálculos de frequência absoluta (n) e relativa (\%), bem como medidas de tendência central (média), sendo os resultados apresentados em forma de tabelas e figuras.

\section{RESULTADO E DISCUSSÃO}

Ao analisar os prontuários da população estudada em relação ao perfil sociodemográfico, observou-se a prevalência do sexo masculino, representada por $56 \%$ dos participantes, seguido do sexo feminino por $44 \%$. Houve o predomínio da faixa etária de 50 a 70 anos com $60 \%$, seguido da faixa etária acima de 70 anos com $40 \%$. Quanto ao estado civil, evidenciou o predomínio de casado(as) $70 \%$, seguidos de viúvos(as) 22\% e solteiro(as) $8 \%$. E sobre o nível de escolaridade, observou-se 0 predomínio de indivíduos com ensino fundamental completo representados por $40 \%$, seguidos do ensino fundamental incompleto e ensino médio completo, ambos com $30 \%$ cada. 
Para um melhor entendimento, os dados de ocupação laboral foram apresentados na figura 1, observando-se prevalência de aposentados, totalizando $46 \%$.

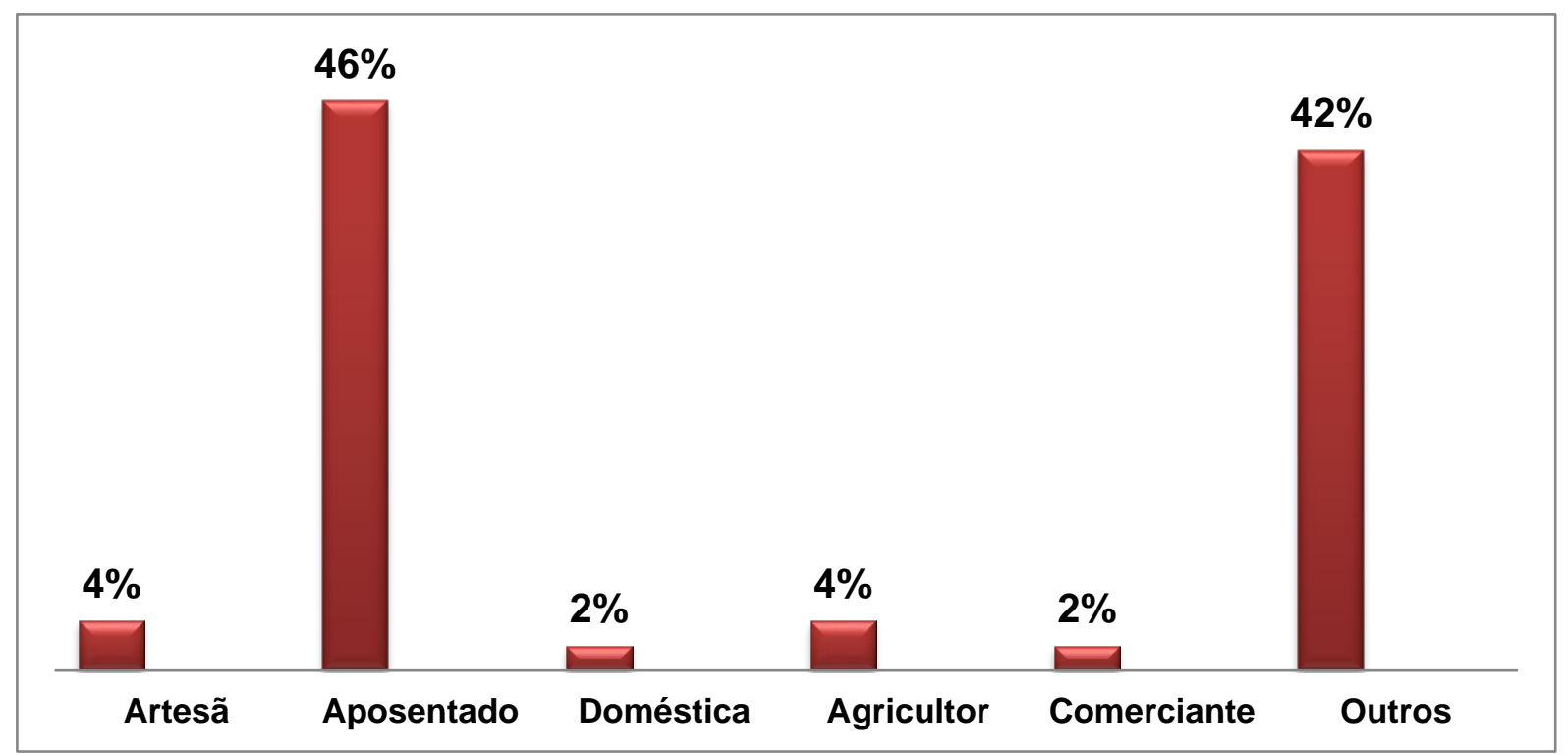

Figura 1 - Distribuição em percentual da ocupação de acordo com prontuários avaliados.

A predominância do sexo masculino encontrada no estudo correlaciona-se aos dados evidenciados por Silveira (2010), em sua pesquisa sobre análise do perfil funcional de pacientes com quadro clínico de AVE, no qual, a representatividade do sexo masculino foi de $63,15 \%$ da amostra.

Grumann et al. (2017) relatam uma maior incidência no sexo feminino, principalmente em idades elevadas, entre 71 a 80 anos, evidenciando que os indivíduos do sexo masculino são acometidos mais precocemente, sendo a incidência mais relevante até os 51 anos, afirmando, assim, que nesse período o homem tem mais chance de ser acometido do que a mulher, e a partir dessa fase os dois gêneros são considerados relevantes, juntamente com outros fatores de risco, para provocar o desencadeamento desta lesão neurológica.

Santos et al. (2012) também evidenciam o sexo feminino como o mais prevalente, e mencionam estudos que revelam uma associação de tal fato a níveis altos de glicemia, uso de contraceptivos orais e idades avançadas, de modo que tais fatores de risco podem colaborar para o desencadeamento dessa patologia. 
Quanto ao estado civil, a maioria era de indivíduos casados(as) e esse dado condiz com o estudo de Damata et al. (2016), ao evidenciar que $75 \%$ dos participantes tinham cônjuges, ressaltando que, diante dos cuidados familiares, há a probabilidade de menores riscos de acometimento cerebral.

Já com base no nível de escolaridade, foi possível evidenciar no presente estudo um baixo índice, corroborando com a pesquisa de Rangel, Belasco e Diccini (2013) que evidenciou um percentual de $59 \%$ dos pacientes avaliados com baixa escolaridade, dado importante, tento em vista que o perfil socioeconômico, quando associado à baixa escolaridade, constitui fator considerável para o desencadeamento do AVE. Tal constatação pode estar relacionada a falta de conhecimento e acesso a informação acerca da doença, bem como a baixa adesão terapêutica e adoção de um estilo de vida inadequado, evidenciado pela má alimentação e falta de exercício físico, de tal modo que indivíduos com formações escolares mantêm sobrevida e um melhor controle dos fatores de risco para as doenças cerebrovasculares.

O estudo evidenciou, quanto ao tipo de AVE, a prevalência do isquêmico, com $92 \%$ dos casos pesquisados, seguidos do tipo hemorrágico com $8 \%$. Tal achado também foi evidenciado nos estudos de Santos et al. (2012), que relataram um predomínio do AVE isquêmico, sendo observado que os fatores de risco impactam significativamente para o desenvolvimento desse quadro.

Corroborando com tais constatações, Costa, Silva e Rocha (2011) evidenciaram um maior acometimento do tipo isquêmico e relatam que o AVE hemorrágico é o menos frequente, entretanto é mais grave em relação ao isquêmico, sendo responsável por causar inúmeras consequências neurológicas.

De acordo com Oliveira (2013) inúmeros fatores influenciam para o desencadear de uma doença, esses que podem ser caracterizados como não modificáveis (idade, raça, etnia e hereditariedade), ou modificáveis (que abrange, principalmente, o estilo de vida como: sedentarismo, alcoolismo, tabagismo; e outros como: hipertensão arterial sistêmica, diabetes mellitus, dislipidemias, fibrilação atrial e estenose carotídea). Ressalta-se, ainda, que os fatores modificáveis podem, na maioria das vezes, ser minimizados quando adotadas mudanças no estilo de vida. 
Segundo Silva (2014) o AVE isquêmico é considerado o mais frequente, ocorrendo em $80 \%$ dos casos, como consequência da formação de trombos e êmbolos, responsáveis pela interrupção do fluxo sanguíneo, provocando a privação de oferta de oxigênio e nutrientes essenciais para o encéfalo, resultando em morte de áreas do encéfalo.

A tabela 1 evidencia o perfil semiológico encontrado, sendo possível observar prevalências maiores para a alteração da marcha, tônus hipotônico e coordenação. Ressalta-se que estão excluídos os valores de normalidade. Quanto ao tônus hipotônico, 20\% dos pacientes apresentavam hipotonia no hemicorpo direito, enquanto $40 \%$ apresentavam hipotonia no hemicorpo esquerdo. Em relação ao tipo hipertônico, $4 \%$ foi o valor encontrado no hemicorpo direito e $8 \%$ no hemicorpo esquerdo.

De acordo com a tabela 1, observa-se um comprometimento no trofismo, sendo evidenciado hipotrofia do lado direito em $10 \%(n=5)$ da amostra estudada, seguido de $8 \%(n=4)$ com hipotrofia do lado esquerdo.

Tabela 1 - Perfil semiológico dos pacientes com acidente vascular encefálico.

\begin{tabular}{llcc}
\hline \multicolumn{1}{c}{ CATEGORIAS } & \multicolumn{1}{c}{ VARIÁVEIS } & $\mathbf{n}$ & $\%$ \\
\hline Tônus Muscular & Hipertônico & 06 & 12 \\
& Hipotônico & $\mathbf{3 0}$ & $\mathbf{6 0}$ \\
Trofismo & Hipotrófico & $\mathbf{0 9}$ & $\mathbf{1 8}$ \\
Sensibilidade & Hipoestesia & $\mathbf{1 1}$ & $\mathbf{2 2}$ \\
Reflexos & Hiperestesia & 05 & 10 \\
& Abolidos & 14 & 28 \\
Coordenação & Diminuídos & $\mathbf{1 5}$ & $\mathbf{3 0}$ \\
Equilíbrio & Alterada & $\mathbf{2 7}$ & $\mathbf{5 4}$ \\
& Estático & 15 & 30 \\
Marcha & Dinâmico & $\mathbf{1 7}$ & $\mathbf{3 4}$ \\
\hline
\end{tabular}

Fonte: Dados da pesquisa, 2019.

O acometimento de formas hipertônica e hipotônica também foi verificado na pesquisa de Grumann et al. (2017) que evidenciou tal achado em um percentual de $50 \%$ dos indivíduos avaliados, de modo que as alterações variavam de indivíduo para indivíduo e relacionavam-se com o grau e localização da lesão. 
Gadelha (2016) relata que o tônus muscular dos indivíduos acometidos se encontra geralmente em estado de hipotonia com duração breve, prosseguindo por horas, dias ou semanas. Em seguida a evolução clínica perpassa para uma fase subaguda, que pode durar de um a três meses. Já a fase crônica consiste na espasticidade do lado oposto a lesão. Já o controle postural estará diminuído no hemicorpo afetado, prejudicando as atividades de vida diária, equilíbrio, postura, bem como, aumentando a probabilidade de quedas, contraturas e deformidades.

Os achados sobre a diminuição do trofismo entram em conformidade com estudo de Melo et al. (2019), ao evidenciar um percentual de $20 \%$ dos pacientes avaliados que apresentavam hipotrofismo, e Batista et al. (2014) ao observar um percentual elevado de hipotrofismo de $15,45 \%$, quando comparado ao hipertrofismo com $1,81 \%$, ou normotrofismo com $6,36 \%$.

O comprometimento muscular pode ocorrer devido à redução no aporte de substratos energéticos necessários para seu bom funcionamento, como também, por modificações diretas na sua fibra muscular, ocasionando redução de resistência e força. Assim sendo, os indivíduos que apresentam alteração de força como consequência da diminuição da massa muscular, podem ter prejuízos na realização das atividades de vida diária (AVD's) e habilidades funcionais (NÓBREGA et al., 2019).

Notou-se que 32\% dos indivíduos apresentavam alterações de sensibilidade, dos quais $22 \%(n=11)$ demonstraram do tipo hipoestesia, e 10\% $(n=5)$ do tipo hiperestesia. Foram acometidos no lado direito 12\% $(n=6)$ em hipoestesia e apenas $4 \%(n=2)$ hiperestesia. Já do lado esquerdo correspondeu 10\% $(n=5)$ em hipoestesia e $6 \%(n=3)$ em hiperestesia.

Esses dados são condizentes com o estudo de Teles; Gusmão (2012) sobre avaliação funcional de pacientes com AVC, composto por 20 indivíduos com diagnóstico de AVE, os quais apresentaram um percentual de $70 \%$ com comprometimento sensorial, relatando, também, que os indivíduos acometidos apresentavam sensibilidade alterada, tanto superficial como profunda.

A maior parte dos indivíduos participantes da presente pesquisa apresentava, ainda, alterações dos reflexos, totalizando 58\% $(n=29)$. Foram encontrados reflexos diminuídos em 30\% $(n=15)$ dos pacientes, com distribuição de 12\% $(n=6)$ para o lado 
direito, $18 \%(\mathrm{n}=9)$ para o lado esquerdo, e com maior acometimento no reflexo patelar, bicipital e tricipital. Os reflexos abolidos se apresentaram em $28 \%(n=14)$, com percentual de $18 \%(n=9)$ no lado direito e $10 \%(n=5)$ lado esquerdo.

Oliveira (2013) afirma que nas lesões neurológicas as alterações na função motora e alterações dos reflexos são achados prevalentes e, junto a eles, surgem a perda do controle postural, paresia e padrões anormais de sinergia, perda da noção de espaço e apraxias, tornando-os inseguros, incapacitados e lentos.

Foi evidenciado um maior acometimento da hemiplegia, de modo que $40 \%$ $(n=20)$ apresentavam hemiplegia à esquerda e $20 \%(n=10)$ à direita, configurando, assim, uma maior prevalência da lesão para o hemisfério direito.

Esse achado condiz com a pesquisa de Sá, Grave e Périco (2014) relatando que $38,4 \%$ dos casos analisados apresentaram hemiplegia à esquerda, enquanto à direita o percentual foi de $26,4 \%$, salientando, ainda, que são manifestações clínicas, quase em sua totalidade, presentes em pessoas com essa patologia.

Woellner et al. (2015) relatam que a hemiparesia é considerada como um dos pontos mais comuns, favorecendo a um maior número de quedas devido ao desequilíbrio, alteração na marcha e diminuição da mobilidade. Já em seu estudo, Grumann et al. (2017) ressaltam que, dos indivíduos acometidos, as mulheres obtiveram um percentual de $70,85 \%$ de hemiparesia subdividida em lado direito e esquerdo, enquanto os homens apresentam um percentual maior de $72,27 \%$.

O estudo evidenciou um percentual considerável de pacientes com comprometimento da compreensão e da expressão, características essas que resultam em falhas no âmbito da memória, dificuldade de comunicar-se, e alteração na capacidade de pronunciar as palavras. Deles, $14 \% \quad(n=7)$ apresentaram comprometimento na compreensão e $12 \%(n=6)$ na expressão.

O estudo de Lima e Maldonado (2016) corrobora com os achados da presente pesquisa acerca da linguagem, ao apontarem essa alteração em 10\% dos indivíduos avaliados, ressaltando a importância da avaliação da linguagem no paciente sequelado por $\mathrm{AVE}$, tendo em vista que a alteração pode desencadear mudanças no comportamento do indivíduo devido à dicção incompreensível. Na interação social, por meio do diálogo, o óbice está ao apresentarem dificuldades na pronúncia de palavras, frases e, até mesmo, em um caso específico, comunicar-se apenas por 
gestos. Viu-se, então, o quanto é importante o tratamento prévio nesses casos, especialmente diante da necessidade de adaptação psicossocial do sequelado em meio à sociedade, que muitas vezes age de forma agressiva e preconceituosa com esses pacientes.

Observou-se nos dados da presente pesquisa que 32 indivíduos dos pacientes apresentavam alteração do equilíbrio dinâmico e estático e que mais da metade dos indivíduos apresentavam coordenação prejudicada.

Os dados são correlatos ao estudo de Messali et al. (2012) sobre avaliação do equilíbrio dinâmico e dificuldades funcionais de indivíduos com sequela de AVE, ao relatarem uma média de $46,23 \%$ de pacientes com alteração do equilíbrio, expondo, ainda, que o controle postural está relacionado com o contato do corpo ao espaço. Relatam que, antes do indivíduo ser acometido, o sistema nervoso central (SNC) funciona por meio de interpretação do que o corpo deseja, assim, o acometimento de áreas do encéfalo vão provocando diversas alterações no SNC, desencadeando complicações que incluem: fraqueza muscular, alteração de tônus e postura, e dificuldade no controle dos movimentos; propiciando, dessa forma, maiores chances de quedas.

Para Lacerda et al. (2018) surge também alteração na função postural, movimentos estereotipados, áreas anestésicas, função comportamental prejudicada, agnosias, apraxias, de modo que há uma dificuldade na realização dos movimentos motores ativamente.

Evidenciou-se nessa pesquisa o comprometimento da marcha em $84 \%$ dos pacientes, com características da marcha ceifante, muitos não deambulavam sem auxílio, sendo a maioria cadeirante. Ressalta-se que a maioria dos pacientes apresentava hemiplegia, hemiparesia e hipotonia de um hemicorpo, fato importante para limitar a marcha fisiológica, deixando o indivíduo incapaz de realizá-la normalmente.

Martins; Magnani (2014) relatam que pacientes hemiplégicos, de certa forma, irão apresentar passos curtos, resultando em uma marcha lenta, falta de controle motor, fraqueza muscular, articulações em posturas como flexão dorsal do pé, limitação da extensão do quadril, movimento excessivo do joelho e amplitude de movimento limitada, dificultando a marcha. 
A análise da amplitude de movimento e da força muscular se configurou como limitação do estudo devido falta de dados concretos nos prontuários avaliados, visto que muitos estavam incompletos e sem coerência. É importante que a avaliação seja bem executada, proporcionando uma coleta de informações que sejam relevantes para estruturar condutas e programas de reabilitação eficazes.

\section{CONCLUSÃO}

O estudo evidenciou o maior comprometimento de indivíduos por AVE do tipo isquêmico, com prevalência no perfil semiológico para as alterações da marcha, tônus hipotônico e alteração da coordenação motor Apresentou, nesses, alteração da mobilidade, desequilíbrio muscular, fraqueza no hemicorpo afetado, postura desalinhada, alteração da sensibilidade, alteração da linguagem e cognitivo comprometido. Quanto ao perfil sociodemográfico, houve a prevalência de pacientes do sexo masculino, com faixa etária entre 50 a 70 anos, casados e com baixa escolaridade.

\section{REFERÊNCIAS BIBLIOGRÁFICAS}

BATISTA, A. J. et al. Perfil epidemiológico do setor de neurologia da clínica escola de fisioterapia da faculdade ingá no ano de 2013. Rev uningá review, v. 17, n. 2, p. 11-15, 2014.

BRASIL. Ministério da Saúde. Conselho Nacional de Saúde. Resolução n. 466, de 12 de dezembro de 2012. Aprova diretrizes e normas regulamentadoras de pesquisas envolvendo seres humanos. Brasília, Diário Oficial da União, 12 dez. 2012.

CARO, C. C.; CRUZ, D. M. C. Correlação entre independência funcional e cognição em homens com AVC. Rev de Terapia Ocupacional da Universidade de São Paulo, v. 28, n. 2, p. 173180, 2017.

COSTA, F. A.; SILVA, D. L. A.; ROCHA, V. M. Estado neurológico e cognição de pacientes pósacidente vascular cerebral. Rev da Escola de Enfermagem da USP, v. 45, n. 5, p. 1083-1088, 2011.

DAMATA, S. R. R. et al. Perfil epidemiológico dos idosos acometidos por acidente vascular cerebral. Rev. Interdisciplinar, v. 9, n. 1, p. 107-117, 2016. 
DELBONI, M. C. C.; MALENGO, P. C; SCHMIDT, E. P. R. Relação entre os aspectos das alterações funcionais e seu impacto na qualidade de vida das pessoas com sequelas de Acidente Vascular Encefálico (AVE). Mundo da Saúde, v. 34, n. 2, p. 165-175, 2010.

FERNANDES, M. B. et al. Independência funcional de indivíduos hemiparéticos crônicos e sua relação com a fisioterapia. Fisioter. Mov., v. 25, n. 2, p. 333-41, 2012.

GADELHA, I. D. S. O comprometimento da capacidade funcional de indivíduos pós-AVC e - acesso aos serviços de fisioterapia. Dissertação - Nível Mestrado do Centro de Ciências Exatas e da Natureza - UFPB. João Pessoa, p. 122. 2016.

GRUMANN, A. R. S. et al. Characteristics of Encephalic Vascular Accident patients treated at a state reference center. Care Journalis Fundamental Online, v. 9, n. 2, p. 315-320, 2017.

LACERDA, I. D. et al. AVE isquêmico em paciente jovem sem fatores de risco: relato de caso. Rev. Med, São Paulo, v. 97, n.3, p. 361-367, 2018.

LEITE, H. R.; NUNES, A.P. N.; CORREA, C. L. Perfil epidemiológico e qualidade de vida dos pacientes acometidos por Acidente Vascular Encefálico. Ar. Ciênc. Saúde UNIPAR, Umuarama, v. 15, n. 1, p. 15-21, 2011.

LIMA, S. M.; MALDONADE, I. Avaliação da linguagem de pacientes no leito hospitalar depois do Acidente Vascular Cerebral. Distúrb Comun, São Paulo, v.28, n.4, p.673-685, dezembro, 2016.

MARTINS, C. R.; MAGNANI, R. M. Análise da marcha de paciente hemiparético decorrente de acidente vascular encefálico: estudo de caso. Trabalho de conclusão de curso (Graduação em Fisioterapia). Universidade Estadual Paulista-UNESP, Marilia/SP, 6f. 2014.

MELO, S. W. A. et al. Funcionalidade e incapacidade dos pacientes pós-acidente vascular encefálico: relato de casos. Rev Pesquisa em Fisioterapia, v. 9, n. 1, p. 101-107, 2019.

MESSALI, F. C. et al. Avaliação do equilíbrio dinâmico e dificuldades funcionais de indivíduos com sequela de acidente vascular encefálico. Colloquium Vitae. v.4, n. especial, p. 245-251, 2012.

NÓBREGA, L. R da et al. Associação da força muscular respiratória e o estado nutricional de pacientes neurológicos. Revista Interdisciplinar em Saúde, v.6, n.1, p. 3-18, abr/jun, 2019.

OLIVEIRA, D. S. Análise do perfil epidemiológico de pacientes com acidente vascular encefálico atendidos na Clínica Escola de Saúde do UNIFOR-MG. Trabalho de Conclusão de Curso (Fisioterapia), Centro Universitário de Formigas-UNIFOR-MG, Formiga, 60 f. 2013.

RANGEL, E. S. S.; BELASCO, A.G.S.; DICCINI, S. Qualidade de vida de pacientes com acidente vascular cerebral em reabilitação. Acta Paulista Enfermagem, v.26, n.2, p.205-12, 2013.

SÁ, B. P.; GRAVE, M. T. Q.; PÉRICO, E. Perfil de pacientes internados por acidente vascular cerebral em hospital do vale do Taquari/RS. Rev Neurocienc, v. 22, n. 3, p. 381-387, 2014.

SANTOS, W. M. et al. Perfil epidemiológicos de pacientes sequelados de acidente vascular cerebral: um estudo transversal. Enciclopédia Biosfera, centro cientifico conhecer, Goiânia, v.8, n.15, p. 1998, 2012.

SILVA, K. S. Análise comparativa da funcionalidade dos membros superiores de hemiparéticos crônicos. Trabalho de Conclusão de Curso (Fisioterapia), Universidade Estadual da Paraíba- (UEPB), Campina Grande - PB, 21 f. 2014. 
SILVEIRA, S. R. Análise do perfil funcional de pacientes com quadro clínico de acidente vascular encefálico (AVE), Ensaios e Ciência: Ciências Biológicas, Agrárias e da Saúde, v.14, n.1, p.15-28, 2010.

TELES, M. S.; GUSMÃO, C. Avaliação funcional de pacientes com Acidente Vascular Cerebral utilizando o protocolo de Fugl-Meyer. Rev Neurociências, v. 20, n. 1, p. 42-49, 2012.

WOELLNER, S. S. et al. Testes de equilíbrio em pacientes hemiparéticos por AVC. Neurociências, v.11, n.1, p.32-40, 2015. 